\title{
NUMERICAL SOLUTION TO THE ACOUSTIC HOMICIDAL CHAUFFEUR GAME
}

\author{
V.S. Patsko \\ Institute of Mathematics and Mechanics \\ S.Kovalevskaya str., 16, Ekaterinburg, 620219, Russia \\ patsko@imm.uran.ru
}

\author{
V.L. Turova \\ Center of Advanced European Studies and Research \\ Friedensplatz 16, 53111 Bonn, Germany \\ turova@caesar.de
}

\begin{abstract}
A well-known differential game in the theory of differential games is the "homicidal chauffeur" problem which was introduced by Isaacs [7]. It is a pursuit-evasion game. In the paper, a variant of this problem proposed by Bernhard [3] is considered. The computation of level sets of the value function in this variant becomes difficult since holes in the "victory domains" of the pursuer can appear. Some results of the computation of level sets of the value function are presented. An explanation of the generation of holes is given, based on the analysis of families of semipermeable curves.
\end{abstract}

\section{INTRODUCTION}

A differential game where an inertial object pursues a non-inertial one is considered. The dynamics of the game is similar to those for the classical $[7,4,11]$ homicidal chauffeur game of $\mathrm{R}$. Isaacs. The difference is that the evader must apply a reduced speed (in order not to be heard by the pursuer) when the distance between him and the pursuer becomes less than a given value. The idea of such a modification was suggested in [3]. The pursuer minimizes the time of capture and the evader maximizes it. The game is over when the evader gets into a given neighborhood of the state of the pursuer (capture neighborhood).

The original version of this chapter was revised: The copyright line was incorrect. This has been corrected. The Erratum to this chapter is available at DOI: 10.1007/978-0-387-35514-6_15

M.J.D. Powell and S. Scholtes (Eds.), System Modelling and Optimization: Methods, Theory and Applications. (O) 2000 IFIP International Federation for Information Processing.

Published by Kluwer Academic Publishers. All rights reserved. 
In [6], level sets of the value function for particular magnitudes of parameters of the problem were computed using an algorithm based on viability theory. The solution to the problem has a complicated structure: holes in the solvability set (in the victory domain) of the pursuer can arise, the evader being safe from the pursuer within these holes.

The investigation of such complex structures of solutions is of great interest for viability theory and the theory of differential games. In the problem considered, the geometry of level sets of the value function differs from the one that was analyzed for other problems with the homicidal chauffeur dynamics $[7,4,11,8,15]$.

In this paper, the problem is studied using an algorithm proposed by the authors for computing level sets of the value function. The algorithm is based on the theory of differential games $[9,10]$. The dependence of the structure of the solution on the parameters of the problem is investigated. The computations are done in the plane because a change of variables can reduce the dimension of the original problem to two [7]. The algorithm uses specific properties of the plane and is very accurate. It allows one to explore some fine peculiarities of the solution. Additionally, the analysis of families of so-called semipermeable curves is used to explain the occurrence of holes.

\section{STATEMENT OF THE PROBLEM}

The dynamics of the game in reduced coordinates has the form $[7,6]$ :

$$
\begin{gathered}
\dot{x}_{1}=-\frac{w^{(1)}}{R} x_{2} \varphi+v_{1}, \quad \dot{x}_{2}=\frac{w^{(1)}}{R} x_{1} \varphi+v_{2}-w^{(1)}, \\
\text { where }|\varphi| \leq 1 \quad \text { and } \quad v \in Q(x) .
\end{gathered}
$$

Here $\left(x_{1}, x_{2}\right)^{\prime}$ is the state vector which gives the relative position of the evader $E$ with respect to the pursuer $P$, and $w^{(1)}$ and $R$ are constants which define the pursuer's velocity and the minimal radius of turn, respectively. The control of player $P$ is $\varphi$, and the control of the evader $E$ is $v=\left(v_{1}, v_{2}\right)^{\prime}$.

The vector $v$ belongs to the circle $Q(x)$ with center at the origin and radius $w^{(2)}(x)=\min \left\{\left(x_{1}^{2}+x_{2}^{2}\right)^{1 / 2}, s\right\} w_{e} / s$, where $w_{e}$ is the maximal value of the velocity of player $E$, and $s$ is a fixed positive number. Thus the radius of the constraint $Q(x)$ on the control of player $E$ is constant and equal to $w_{e}$ outside the circle of radius $s$ with center at the origin, but the radius is proportional to $|x|$ inside this circle.

The terminal set $M$ is the rectangle $\left\{\left(x_{1}, x_{2}\right) \in R^{2}:-3.5 \leq x_{1} \leq 3.5\right.$, $\left.-0.2 \leq x_{2} \leq 0\right\}$. The objective of the control $\varphi$ is to minimize the time of attaining the terminal set $M$, but the objective of the control 
$v=\left(v_{1}, v_{2}\right)^{\prime}$ is to maximize this time. Therefore the payoff of the game is the time of attaining the terminal set.

The statement of the problem was taken from [6]. In the classical statement [7] of the problem, the terminal set (capture neighborhood) is a circle. A circle can be used in the acoustic version too. However, more interesting cases from the mathematical point of view arise when the capture neighborhood is a rectangle with its horizontal side much greater than its vertical side.

The game is treated in frames of formalization from $[9,10]$. We are interested in finding level sets $W(T, M), T>0$. Each of them is the set of all initial states $x_{0}$ in the plane such that player $P$ can guarantee the transition of the state vector to the set $M$ within time $T$.

\section{THE ALGORITHM}

Here the main idea of the algorithm for computing the level sets $W(T, M)$ of the value function is described.

Let $\Delta$ be a time step of the backward procedure. Let the $i$-th level set of the value function, namely $W(i \Delta, M)$, be available. This is the maximal set from where the pursuer $P$ guarantees the termination of the game within the time $i \Delta$. On the basis of this set, we compute the set $W((i+1) \Delta, M)$, consisting of all states from which player $P$ guarantees the attainment of $W(i \Delta, M)$ within time $\Delta$. As a result of such computations for $i=0,1,2, \ldots$, we obtain the collection of embedded sets $W(\Delta, M) \subset W(2 \Delta, M) \subset \cdots \subset W(i \Delta, M) \subset \cdots \subset W(T, M)$.

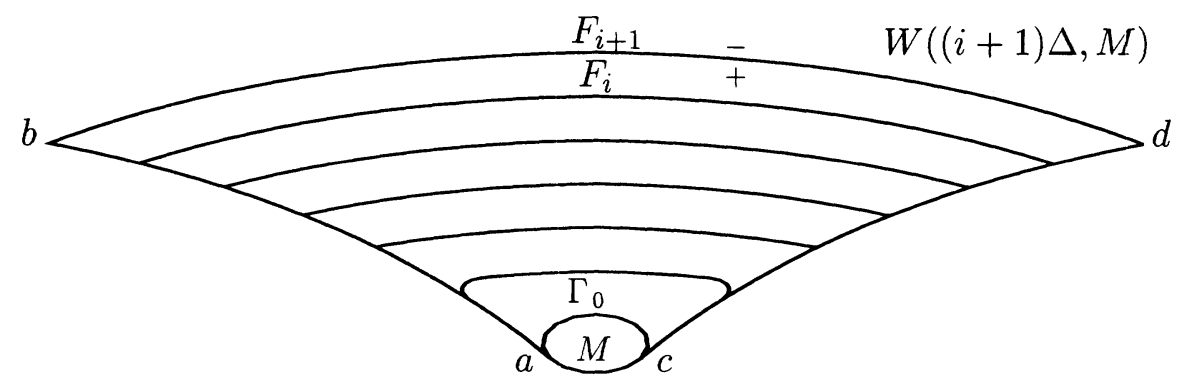

Figure 1 Construction of the sets $W(i \Delta, M)$

This is a dynamic programming method. In the theory of differential games, the fundamental idea of the backward construction of level sets was considered in works of Isaacs, Fleming, Pontryagin, Krasovskii and Pschenichnyi. 
The central part of our algorithm is the notion of a front. The front $F_{i+1}$ contains all points on the boundary of the set $W((i+1) \Delta, M)$ with the property that the minimal guaranteed time of attaining the previous set $W(i \Delta, M)$ is precisely $\Delta$. The side of the front in the backward time direction will be called negative, and the opposite side will be called positive, as in Figure 1. The algorithm computes a new front $F_{i+1}$ using the previous front $F_{i}$. For the first step of the backward procedure, $F_{0}$ coincides with the usable part [7] $\Gamma_{0}$ of the boundary of $M$. The barrier lines are obtained via connection of the corresponding ends of the fronts. In Figure 1, the lines $a b$ and $c d$ are barriers.

We explain briefly how the fronts are constructed. Using the notation $p(x)=\left(-x_{2}, x_{1}\right)^{\prime} \cdot w^{(1)} / R$ and $g=\left(0,-w^{(1)}\right)^{\prime}$, we rewrite the equations (1) as $\dot{x}=p(x) \varphi+v+g$. In the computation, each front is stored as an ordered collection of points, so fronts are polygonal lines. An apex of a polygonal line is called a point of local convexity if the angle between the positive sides of the adjoining links is less than $\pi$. An apex of a front is a point of local concavity if the above angle is greater than $\pi$. A cone $K$ of outer (inner) normal vectors is assigned to each point of local convexity (concavity). At the endpoints of the front, the cone $K$ is defined in a different way: one extreme ray of the cone is the outer normal vector to the front link, and the other extreme ray of the cone is defined by some special relations. For each fixed point $x_{*} \in F_{i}$ of local convexity and any vector $\ell \in K\left(x_{*}\right)$, the extremal controls $\varphi^{\circ}, v^{\circ}$ take the values $\varphi^{\circ}=\operatorname{argmin}\left\{\ell^{\prime} p\left(x_{*}\right) \varphi:|\varphi| \leq 1\right\}$ and $v^{\circ}=\operatorname{argmax}\left\{\ell^{\prime} v: v \in Q\left(x_{*}\right)\right\}$. Similarly, for the points of local concavity, the extremal controls are $\varphi^{\circ}=\operatorname{argmax}\left\{\ell^{\prime} p\left(x_{*}\right) \varphi:|\varphi| \leq 1\right\}$ and $v^{\circ}=\operatorname{argmin}\left\{\ell^{\prime} v: v \in Q\left(x_{*}\right)\right\}$.

The extremal control $\varphi^{\circ}$ of player $P$ can switch its value from one extreme value to another, not only at the apexes of the front, but also at inner points of the front links. In the game considered, such a switching occurs at not more than one inner point of each front link, due to the linearity of the dynamics in $x$ and $\varphi$. The points where such a switching takes place will be called neutral. The collection of all neutral points is included (with the preservation of ordering) in the collection of apexes defining the front. Each neutral point divides the original link into two parts that are also considered as links of the front.

Other additional division points on the front links may also be introduced, which take into account the dependence of the constraint on the control of player $E$ on $x$. The cone $K$ for a neutral or additional point contains only the outer normal vector at the point. Further, the extremal controls of the players are given by the above formulae for the local convexity case. 
Using the extremal controls, one computes the extremal trajectory $x(\sigma)=x_{*}-\sigma\left(p\left(x_{*}\right) \varphi^{\circ}+v^{\circ}+g\right), \sigma \in(0, \Delta]$, in reverse time. If the extremal controls are not unique at $x_{*}$, a bundle of extremal trajectories emanating from the point $x_{*}$ is considered.

As $x_{*}$ ranges through $F_{i}$, the ends of the extremal trajectories at $\sigma=\Delta$ are used to form the next front $F_{i+1}$. One can divide $F_{i}$ into regular parts so that the extremal trajectories emanating from the points of one part do not intersect for $\sigma \in(0, \Delta]$. Thus each regular part generates a regular field of extremal trajectories. The ends of these trajectories form an ordered collection of points. Being connected, these points give a polygonal line, which is called the secondary arc. The new front $F_{i+1}$ is obtained by processing the regular secondary arcs, the processing being reduced to the intersection of secondary arcs.

We consider this procedure for the simple case shown in Figure 2. Here the front $F_{i}$ consists of two regular parts $\left[z_{1} \cdots z_{\omega}\right]$ and $\left[z_{\omega} \cdots z_{r}\right]$. Both parts are composed of local convexity points. The ends of the extremal trajectories computed at $\sigma=\Delta$ give two secondary arcs, namely $\left[\xi_{1} \xi_{2} \cdots \xi_{s}\right]$ and $\left[\xi_{s+1} \cdots \xi_{m}\right]$, as shown in the left half of the figure. The control of player $E$ can be chosen for each of the points $\xi_{s}$ and $\xi_{s+1}$ so that the trajectories of the system (1) cannot reach the front $F_{i}$ within time $\Delta$. Therefore, the "swallow tail" $\xi_{s} \xi_{\alpha} \xi_{s+1}$ is not included in the front $F_{i+1}=\left[\xi_{1} \xi_{2} \ldots \xi_{\alpha} \ldots \xi_{m}\right]$, which is drawn on the right hand side of Figure 2.

a)

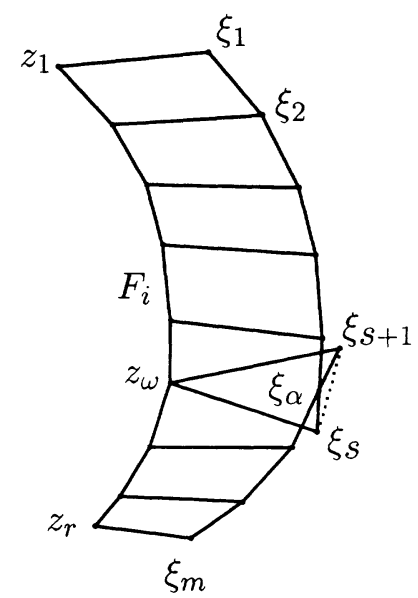

b)

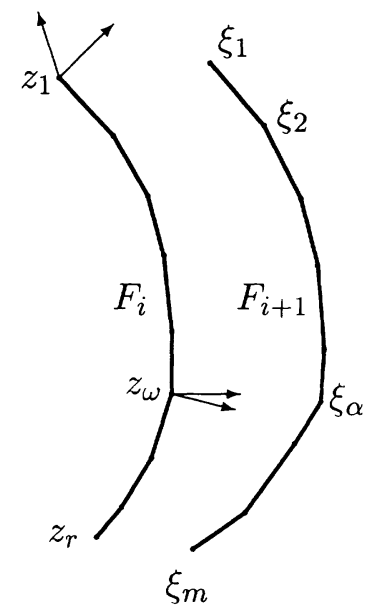

Figure 2 Construction of fronts 
Unfortunately, very often, it is not sufficient to intersect neighboring secondary arcs only. In Figure 3, for example, the secondary $\operatorname{arcs} S_{1}, S_{2}$ and $S_{3}$ are computed sequentially, but the next front is obtained due to the intersection of $S_{1}$ and $S_{3}$.

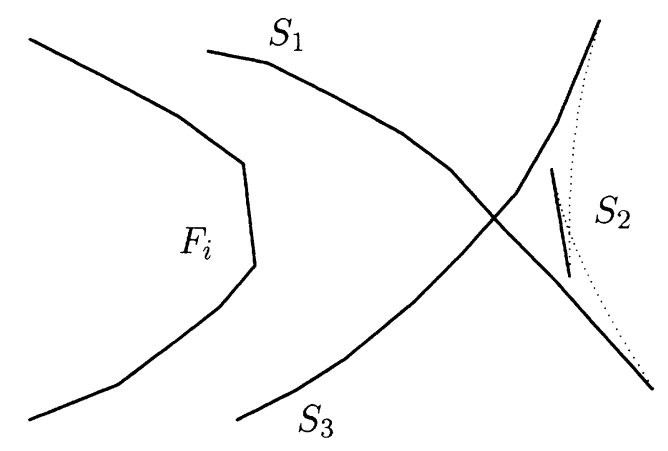

Figure 3 Secondary arcs: complicated case of disposition

Thus the algorithm produces a collection of fronts. In the course of computations, possible self-intersections of fronts and their collisions with the barrier lines are processed. The details of the algorithm can be found in $[12,13,14]$.

\section{SEMIPERMEABLE CURVES}

In this section, the results of some analysis of families of semipermeable curves in differential games with homicidal chauffeur dynamics will be given. Using these results, one can find the solvability sets of the game of kind [7]. Since the set $W(T, M)$ converges to the solvability set of the corresponding game of kind as $T \rightarrow \infty$, solutions to this game of kind can be used for verifying the computation of the sets $W(T, M)$. The families of semipermeable curves can also be helpful for checking the computations of level sets of the value function within solvability sets.

The families of semipermeable curves are determined from only the dynamics of the system and the bounds on the controls of the players. We explain now what semipermeable curves mean. Let

$$
\begin{aligned}
H(\ell, x) & =\min _{\varphi \in[-1,1]} \max _{v \in Q(x)} \ell^{\prime} f(x, \varphi, v) \\
& =\max _{v \in Q(x)} \min _{\varphi \in[-1,1]} \ell^{\prime} f(x, \varphi, v), \quad x \in R^{2}, \quad \ell \in R^{2},
\end{aligned}
$$


be the Hamiltonian of the game. Here $f(x, \varphi, v)=p(x) \varphi+v+g$. It is easy to see that the function $\ell \rightarrow H(\ell, x)$ is convex in $\ell$ in the cones $\ell^{\prime} p(x) \geq 0$ and $\ell^{\prime} p(x) \leq 0$ for any fixed $x \in R^{2}$. Fix $x$ and consider $\ell$ such that $H(\ell, x)=0$. Letting $\varphi^{*}=\operatorname{argmin}\left\{\ell^{\prime} p(x) \varphi: \varphi \in[-1,1]\right\}$ and $v^{*}=\operatorname{argmax}\left\{\ell^{\prime} v: v \in Q(x)\right\}$, it follows that $\ell^{\prime} f\left(x, \varphi^{*}, v\right) \leq 0$ holds for any $v \in Q(x)$, and $\ell^{\prime} f\left(x, \varphi, v^{*}\right) \geq 0$ holds for any $\varphi \in[-1,1]$. This means that the direction $f\left(x, \varphi^{*}, v^{*}\right)$, which is orthogonal to $\ell$, separates the vectograms $U\left(v^{*}\right)=\left\{f\left(x, \varphi, v^{*}\right): \varphi \in[-1,1]\right\}$ and $V\left(\varphi^{*}\right)=$ $\left\{f\left(x, \varphi^{*}, v\right): v \in Q(x)\right\}$ of the players $P$ and $E$ as in Figure 4. Such a direction is called semipermeable. A smooth curve is called a semipermeable curve if the tangent vector at any point of this curve is a semipermeable direction.

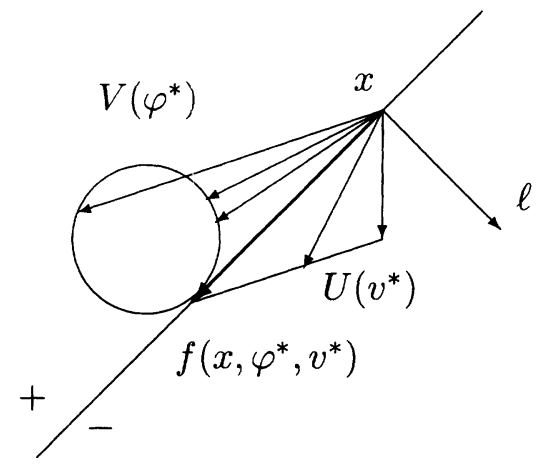

Figure 4 Semipermeable direction

We now describe how the families of semipermeable curves can be obtained. The semipermeable directions are derived from the roots of the equation $H(\ell, x)=0$. We distinguish the roots "-" to "+" and the roots "+" to "-". When classifying these roots, we suppose that $\ell \in \mathcal{E}$, where $\mathcal{E}$ is the boundary of a convex polygon containing the origin. We say that $\ell_{*}$ is a root - to + if $H\left(\ell_{*}, x\right)=0$, and if $H(\ell, x)<0$ $(H(\ell, x)>0)$ for $\ell<\ell_{*}\left(\ell>\ell_{*}\right)$ that are sufficiently close to $\ell_{*}$, where the notation $\ell<\ell_{*}$ means that the direction of the vector $\ell$ can be obtained from the direction of the vector $\ell_{*}$ using a counterclockwise rotation through an angle not exceeding $\pi$. The roots - to + and the roots + to - are called roots of the first and second type, respectively. Due to the above mentioned property of the piecewise convexity of the function $H(\cdot, x)$, the equation $H(\ell, x)=0$ can have at most two roots of each type for any given $x$. 
Let us denote the roots by $\ell^{(j), i}(x)$. The left index corresponds to the type of root ( - to + or + to - ). The right index takes the value 1 or 2 , and indicates whether the minimum in (2) occurs for $\varphi=1$ or $\varphi=-1$.

One can find the domains of the functions $\ell^{(j), i}(\cdot)$. They have very simple structures for the classical formulation of the homicidal chauffeur problem. In this case, the constraint $Q$ on the control of player $E$ does not depend on $x$, so we have $w^{(2)}=w_{e}$.

Figure 5 shows the domains of $\ell^{(j), i}(\cdot)$ in the classical case $w^{(2)}=$ $w_{e} \leq w^{(1)}$. Two symmetric cones with a joint apex at the origin are cut by polygonal approximations to circular $\operatorname{arcs}$ of radius $w^{(2)} R / w^{(1)}$, the centers of the arcs being at the points $(-R, 0)$ and $(R, 0)$. The regions of values of $x$ where two roots of each type exist are marked $A$ and $B$ in the figure. There is only one root of each type at the points $x$ that are outside $A$ and $B$.

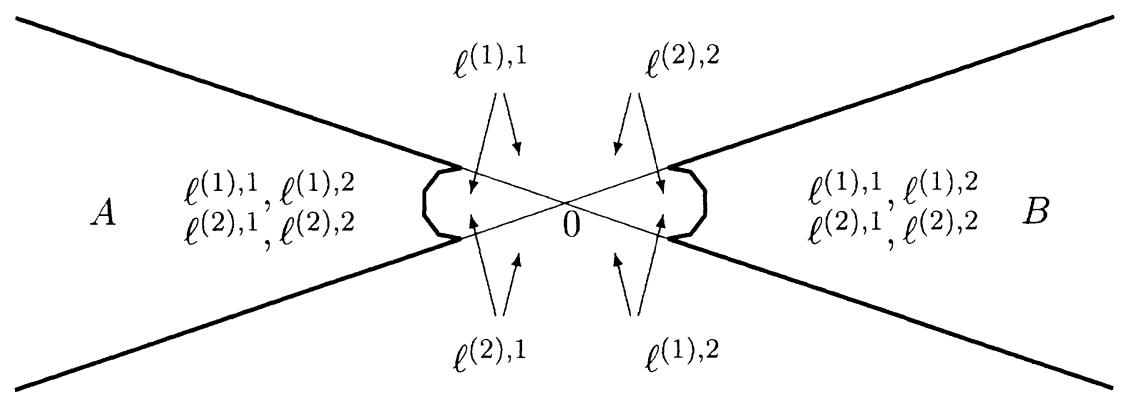

Figure 5 Domains of the functions $\ell^{(j), i}(\cdot)$ when $w^{(2)}=w_{e} \leq w^{(1)}$

In Figure 6, the domains of $\ell^{(j), i}(\cdot)$ are depicted for $w^{(2)}=w_{e}>$ $w^{(1)}$. The digits 4,2 and 0 state the number of roots. In this case, a region $C$ (the intersection of the circles of radius $w^{(2)} R / w^{(1)}$ with centers at $(-R, 0)$ and $(R, 0))$ occurs where roots do not exist. The following property holds true for any point $x \in C$ : for any $\varphi \in[-1,1]$ there exists $v \in Q$ such that $f(x, \varphi, v)=0$. Therefore, in the region $C$, player $E$ can counter any control of player $P$, so the state remains immovable all the time. Further, if a point $x$ with the above property does not belong to the terminal set $M$, then $M$ cannot be reached from $x$. Regions of such points are called the superiority sets of player $E$.

Using the forms of the domains of $\ell^{(j), i}(\cdot)$ in the classical case, one can construct the domains for the case when $Q$ depends on $x$. We describe schematically how it can be done. First note that $w^{(2)}$ is constant on the circumference of any fixed circle with center $(0,0)$. Further, $w^{(2)}=w_{e}$ 


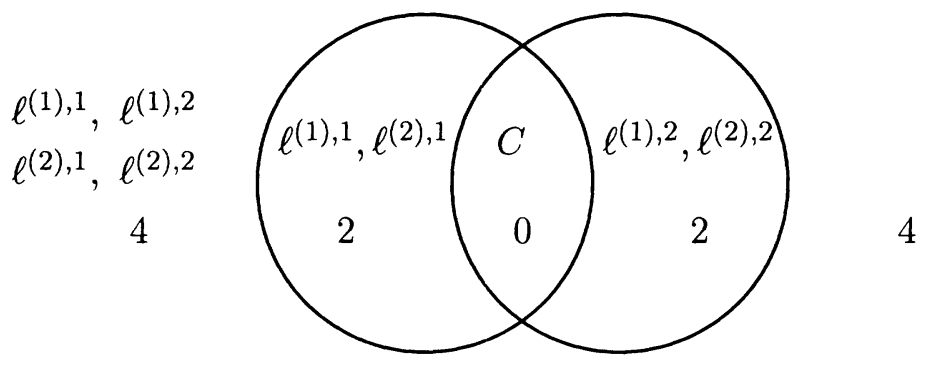

Figure 6 Domains of the functions $\ell^{(j), i}$ when $w^{(2)}=w_{e}>w^{(1)}$.

holds outside the circle of radius $s$. Let $\Omega(r)$ be the circumference of the circle of radius $r$ with center at $(0,0)$. Find $w^{(2)}(r)=\min \{r, s\} w_{e} / s$. If $w^{(2)}(r) \leq w^{(1)}$, then put the points $x \in \Omega(r)$ onto the domains of Figure 5 constructed for $w^{(2)}=w^{(2)}(r)$. Otherwise, if $w^{(2)}(r)>w^{(1)}$, then put these points onto the domains of Figure 6 . Thus a division of $\Omega(r)$ into arcs is obtained. The number and the type of roots are the same for all points of each arc. In Figure 7, the division points $a, b, c$ and $d$, and those symmetric to them in the left half-plane, are shown, $\Omega(r)$ being the dotted line. In Figure 8, the division points $e$ and $f$, and those symmetric to them, are depicted.

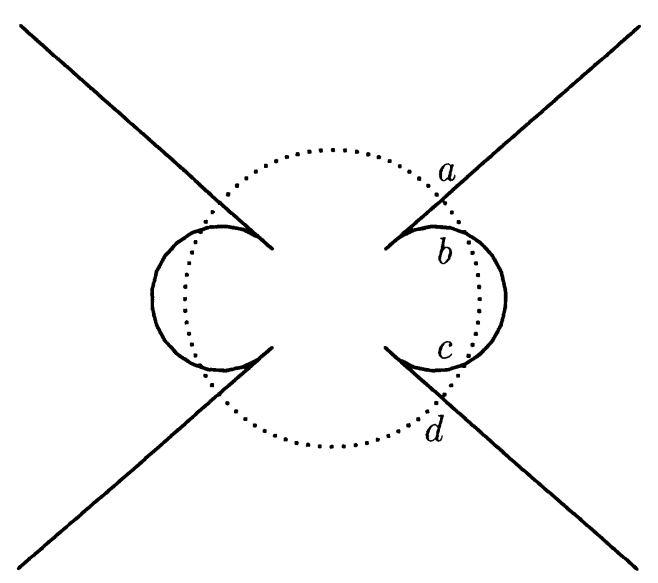

Figure 7 Construction of domains of $\ell^{(j), i}(\cdot)$ when $w^{(2)}(r) \leq w^{(1)}$ 
This technique is applied for every $r$ in $[0, s]$, and identically named division points are connected. Thus the circle of radius $s$ is divided into parts according to the kinds of roots. Outside this circle, the dividing lines coincide with the lines constructed for the case when $Q$ does not depend on $x$. We use the lines of Figure 5 or Figure 6 , depending on $w_{e} \leq w^{(1)}$ or $w_{e}>w^{(1)}$, respectively.

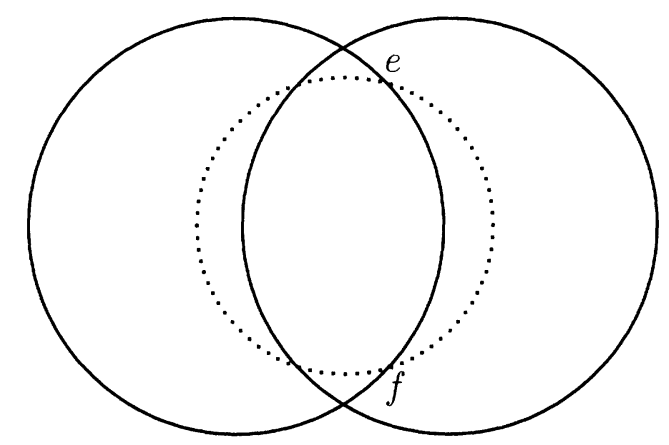

Figure 8 Construction of domains of $\ell^{(j), i}(\cdot)$ when $w^{(2)}(r)>w^{(1)}$

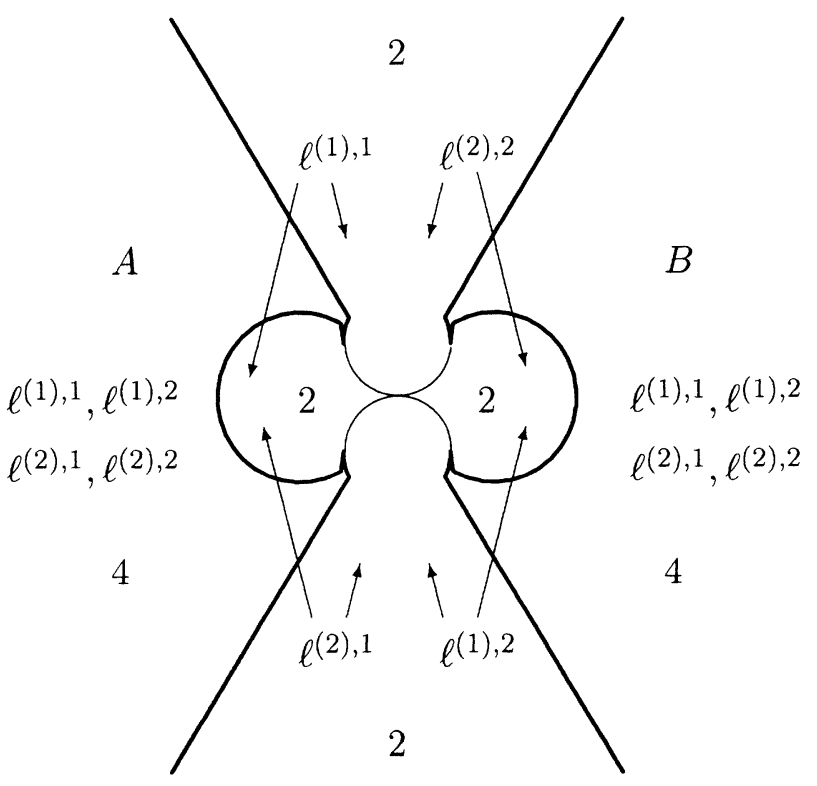

Figure 9 Domains of the functions $\ell^{(j), i}(\cdot)$ when $Q$ depends on $x$ and $w_{e}=0.8$ 


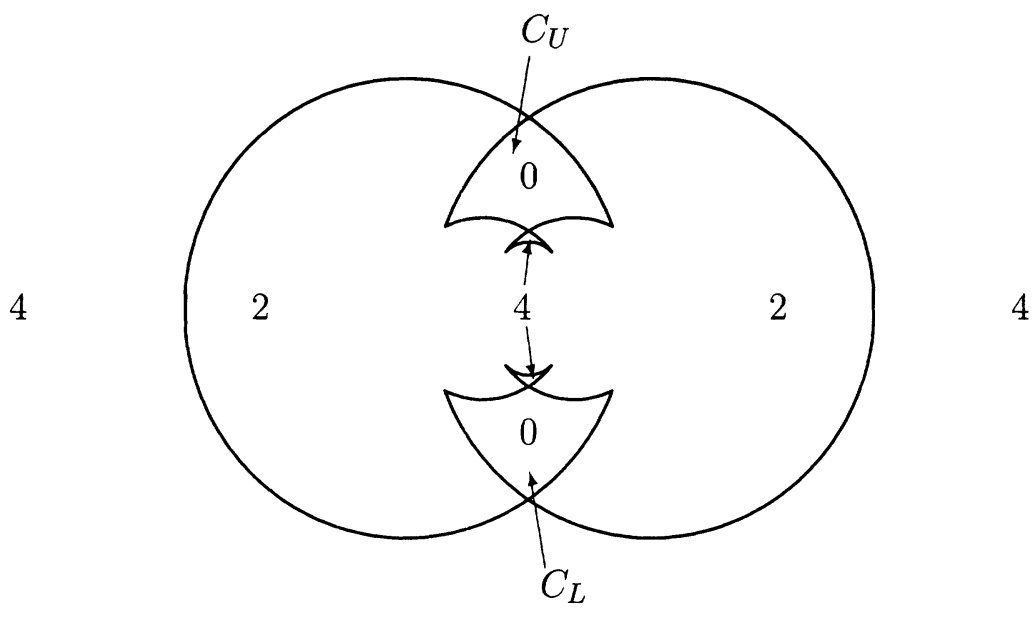

Figure 10 Superiority sets of player $E$ when $Q$ depends on $x$ and $w_{e}=1.8$

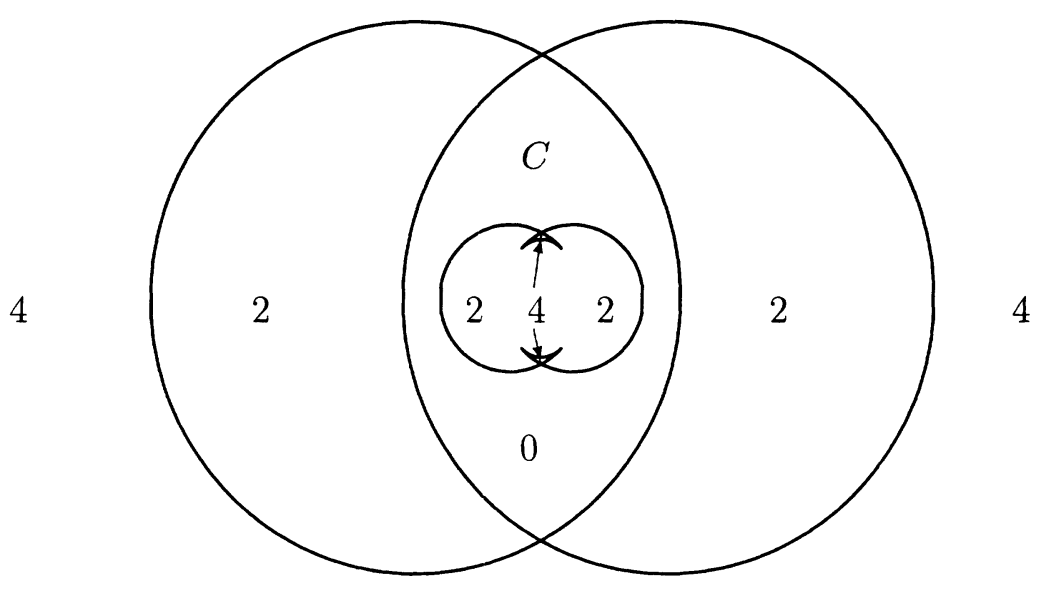

Figure 11 Superiority set of player $E$ when $Q$ depends on $x$ and $w_{e}=2$

Figures 9,10 and 11 were constructed in this way for the parameters $w^{(1)}=1, R=0.8, s=0.75$ and $w_{e}=0.8,1.8$ and 2. In Figure 9, the domains of the functions $\ell^{(j), i}(\cdot)$ are shown, and also the sets that are analogous to $A$ and $B$ in Figure 5 are marked. In Figure 10, two symmetric superiority sets of player $E$ arise, the upper set being denoted by $C_{U}$ and the lower set by $C_{L}$. If we increase $w_{e}$, the sets $C_{U}$ and $C_{L}$ 
expand and form the double connected region that is denoted by $C$ in Figure 11. The number of roots of the equation $H(\ell, x)=0$ is also given in Figures 10 and 11.

Figure 12 shows a fragment of the central part of Figure 10. The lines that separate the domains of the functions $\ell^{(j), i}(\cdot)$ are included.

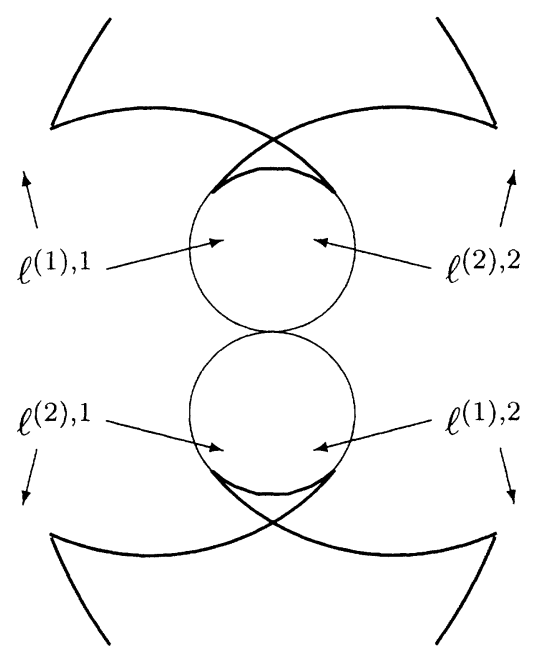

Figure 12 Domains of the functions $\ell^{(j), i}$ in part of Figure 10

The function $\ell^{(j), i}(\cdot)$ is Lipschitz continuous on any closed bounded subset of the interior of its domain. We consider the two-dimensional differential equation

$$
d x / d t=\Pi \ell^{(j), i}(x)
$$

where $\Pi$ is the matrix of rotation through the angle $\pi / 2$, the rotation being clockwise or counterclockwise if $j=1$ or $j=2$, respectively. Since the tangent vector at each point of the trajectory defined by this equation is a semipermeable direction, the trajectories are semipermeable curves. Therefore player $P$ can keep the state vector $x$ on one side of the curve (positive side), and player $E$ can keep $x$ on the other (negative) side. Further, equation (3) specifies a family $\Lambda^{(j), i}$ of semipermeable curves, such that a unique smooth semipermeable curve goes through each point $x$ of the domain of $\ell^{(j), i}(\cdot)$, the root $\ell^{(j), i}(x)$ being the normal vector to the curve at the point $x$. The notation $p^{(j), i}$ will be used for the curves of the family $\Lambda^{(j), i}$.

The family $\Lambda^{(1), 1}$ for the values of the parameters of Figure 10 is depicted in Figure 13. The arrows show the direction of motion in reverse 


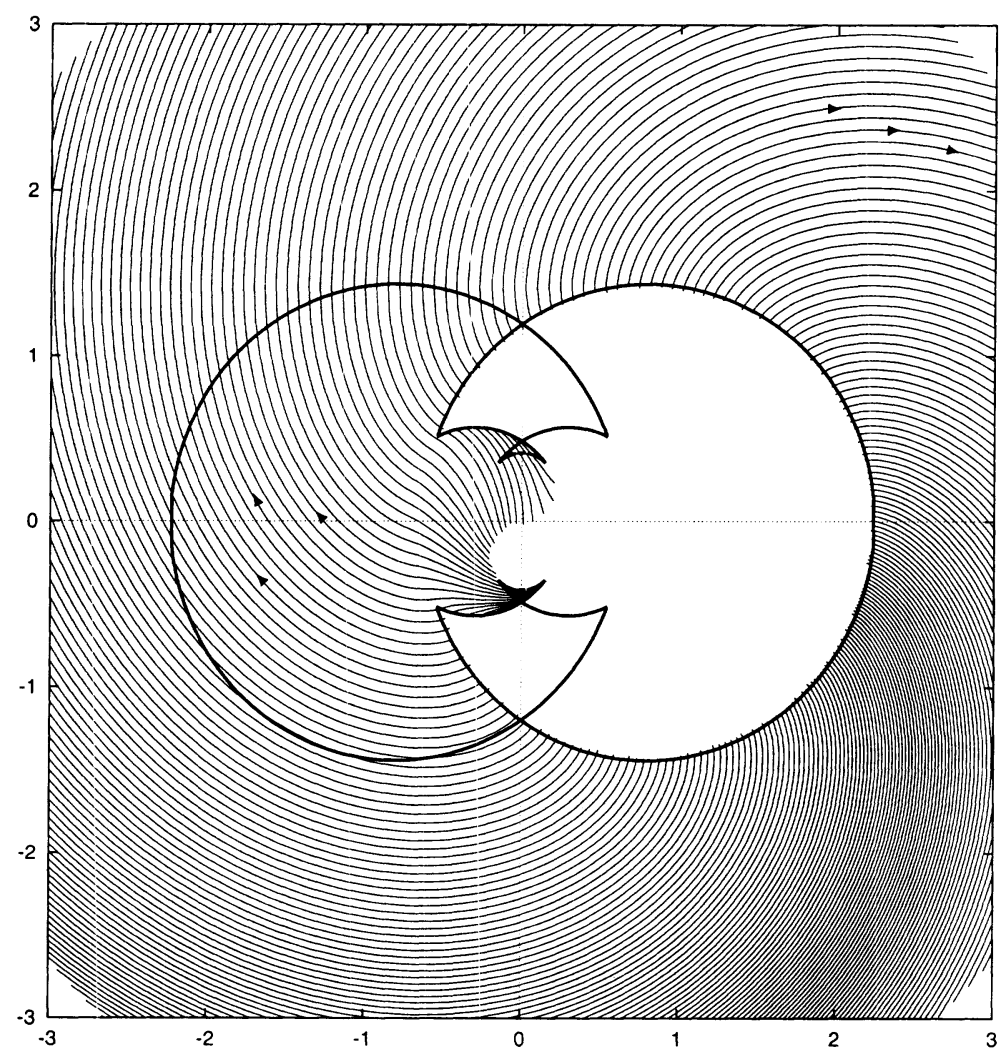

Figure 13 Family of semipermeable curves for the root $\ell^{(1), 1}$

time. The families $\Lambda^{(1), 2}, \Lambda^{(2), 1}$ and $\Lambda^{(2), 2}$ can be obtained from $\Lambda^{(1), 1}$ by reflections in the $x_{1}$ - and $x_{2}$-axes.

\section{SUPERIORITY SETS}

In this section, the role of superiority sets in the appearance of holes within the solvability sets will be explained. As noted above, there can be one doubly connected superiority set $C$ of player $E$, or two simply connected sets $C_{U}$ and $C_{L}$, or the superiority set can be empty.

Let $D$ be a connected superiority set of player $E$, and let the objective of this player be to bring the state of the system to the set $D$. Denote by $D^{*}$ the maximal solvability set (victory domain) of player $E$. It follows from the definition of $D^{*}$ that $E$ can bring the state of the system to $D$ from any point $x \in D^{*}$, but player $P$ can prevent the state of the system from approaching the set $D$ for any point $x \notin D^{*}$. Since $D$ is 
a superiority set of $E$, it possesses the property of $v$-stability $[9,10]$ (or viability for $E[1,6]$ ), and the set $D^{*}$ is $v$-stable too. This means that player $E$ can hold the trajectories of the system in $D^{*}$ for infinite time. Hence, if $D^{*} \cap M=\emptyset$, then the time for achieving the terminal set $M$ in the main problem is infinite for any $x$ in $D^{*}$.

The boundary of $D^{*}$ is composed of smooth semipermeable curves of the families $\Lambda^{(j), i}$. The joins of these curves are called "sewing points", and they possess the semipermeability property [5]. In some cases, a part of the boundary of $D^{*}$ can coincide with a part of the boundary of $D$.

Due to the simple geometry of the sets $D$ of the problem considered, the sets $D^{*}$ can be obtained easily using the families of semipermeable curves. For example, Figure 14 shows the configuration of $D^{*}$ when $D=C_{U}$ as in Figure 10. The sewing point of the curves $p^{(2), 2}$ and $p^{(1), 2}$, and the symmetric sewing point of the curves $p^{(1), 1}$ and $p^{(2), 1}$, lie on the boundary of $D$.

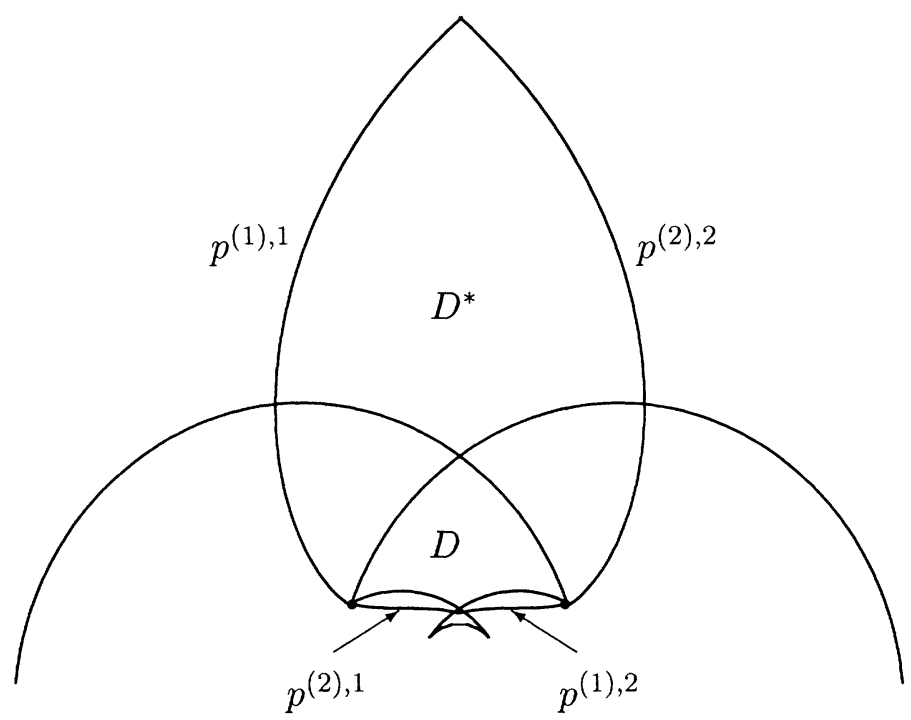

Figure 14 Generation of the hole $D^{*}$ due to the superiority set $D$

Since level lines of the value function do not "penetrate" into the sets $D^{*}$ in the case $D^{*} \cap M=\emptyset$, one can easily generate examples where holes occur in the solvability sets, using this knowledge of the geometry of the sets $D^{*}$. 


\section{COMPUTATIONAL RESULTS}

In this section, the dependence of the solution on the parameter $w_{e}$ is demonstrated. Other parameters of the problem are fixed and have the values $w^{(1)}=1, R=0.8$ and $s=0.75$. The circle $Q(x)$ is approximated by a polygon. Let $\tau$ be the reverse time in the backward procedure for the construction of fronts. The optimal time for a given state $x$ is the least time $\tau$ such that $x \in W(\tau, M)$.

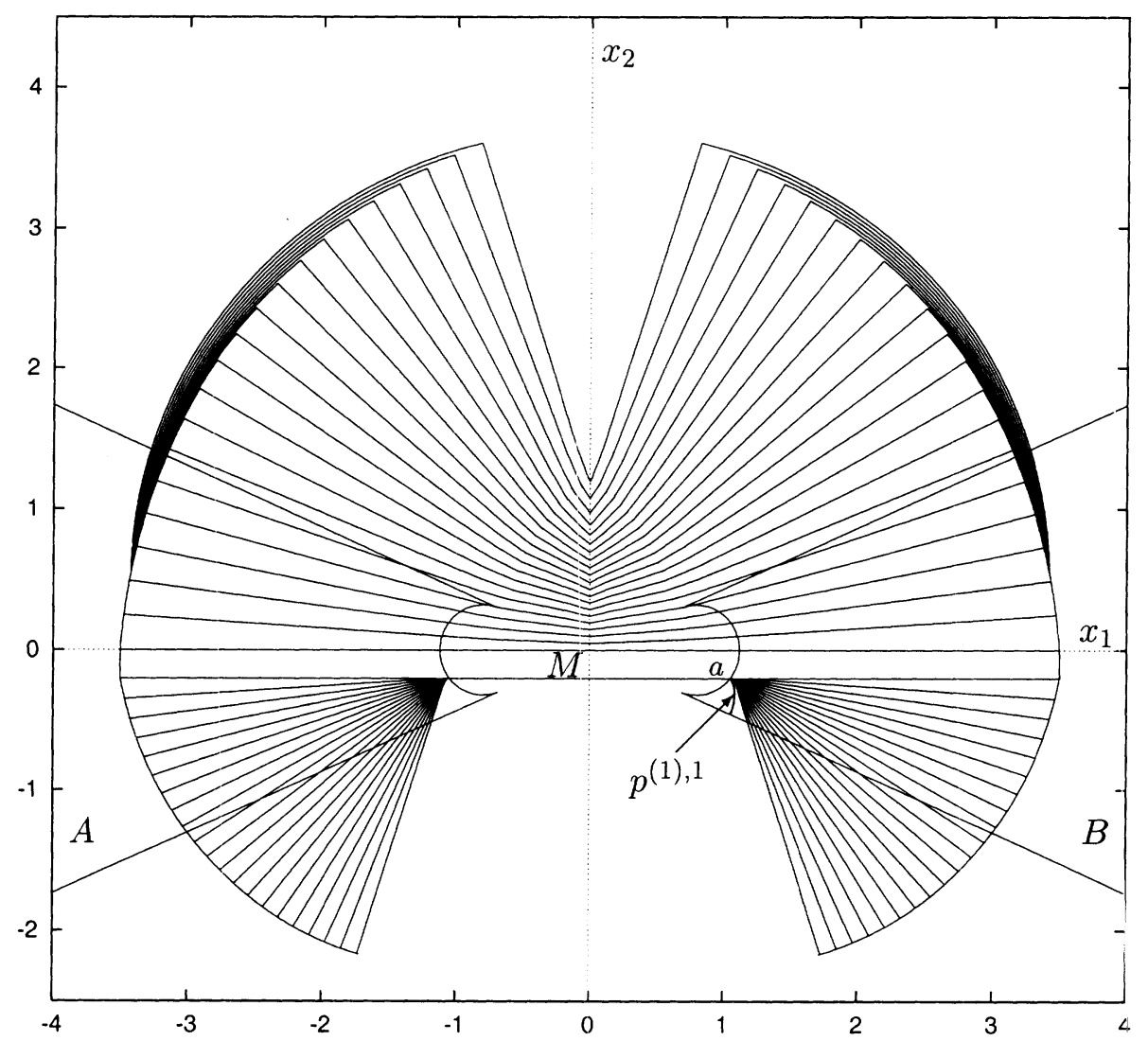

Figure 15200 upper and lower fronts for $w_{e}=0.4$ (every 10th front is plotted)

In Figure 15, the initial computations for $w_{e}=0.4$ are shown. The step $\Delta$ is 0.005 . The usable part of the terminal set $M$ consists of three segments: the upper side of $M$ and two segments on the lower side. The upper fronts that occur until $\tau=0.29$ are bounded on the left and right by barrier lines. At $\tau=0.29$, these barrier lines meet the upper boundaries of the sets $A$ and $B$ (see Figure 9), so they terminate. The value function is discontinuous across the barrier lines. For $\tau>0.29$, the 
fronts begin to envelop the barrier lines, and left and right corner points arise. The propagation of the front beyond the barrier lines from these corner points is at a very low rate. An enlargement of this development of the fronts on the right hand side is presented in Figure 16.

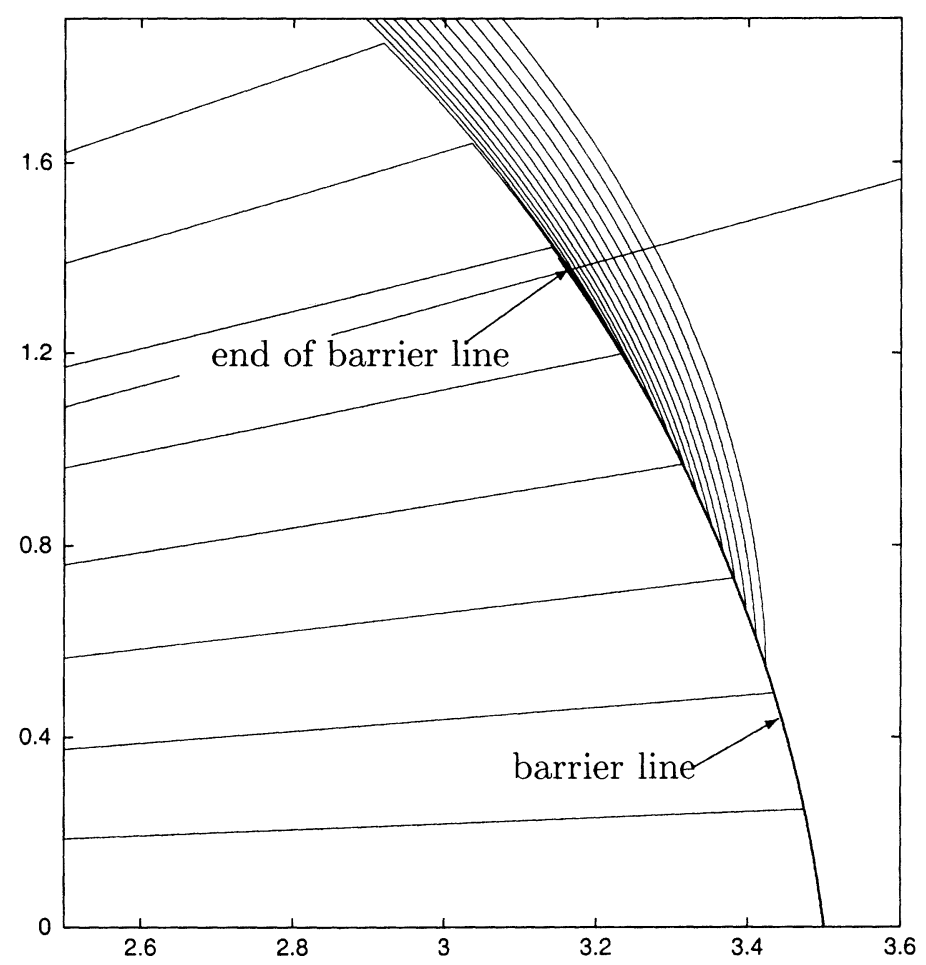

Figure 16 The structure of fronts near the barrier line

The continuation of the computation is shown in Figure 17. The upper and lower fronts are calculated until $\tau=1.6$ and $\tau=3.3$, respectively. The left and right lower fronts collide at $\tau=1.76$. Only one lower front remains after this collision. The greatest value of $\tau$ below $M$ occurs on the lower boundary of $M$ at the point $(0,-0.2)$.

An enlargement of the accumulation of the lower fronts is shown in Figure 18. We see that the end of the front moves along the terminal set from the end of the usable part to the point $a$ on the boundary of the set $B$. The accumulation of fronts begins when they approach the semipermeable curve $p^{(1), 1}$ that emanates from the point $a$, as shown in Figure 15 . The value function changes very rapidly in the accumulation region, but it remains continuous. 


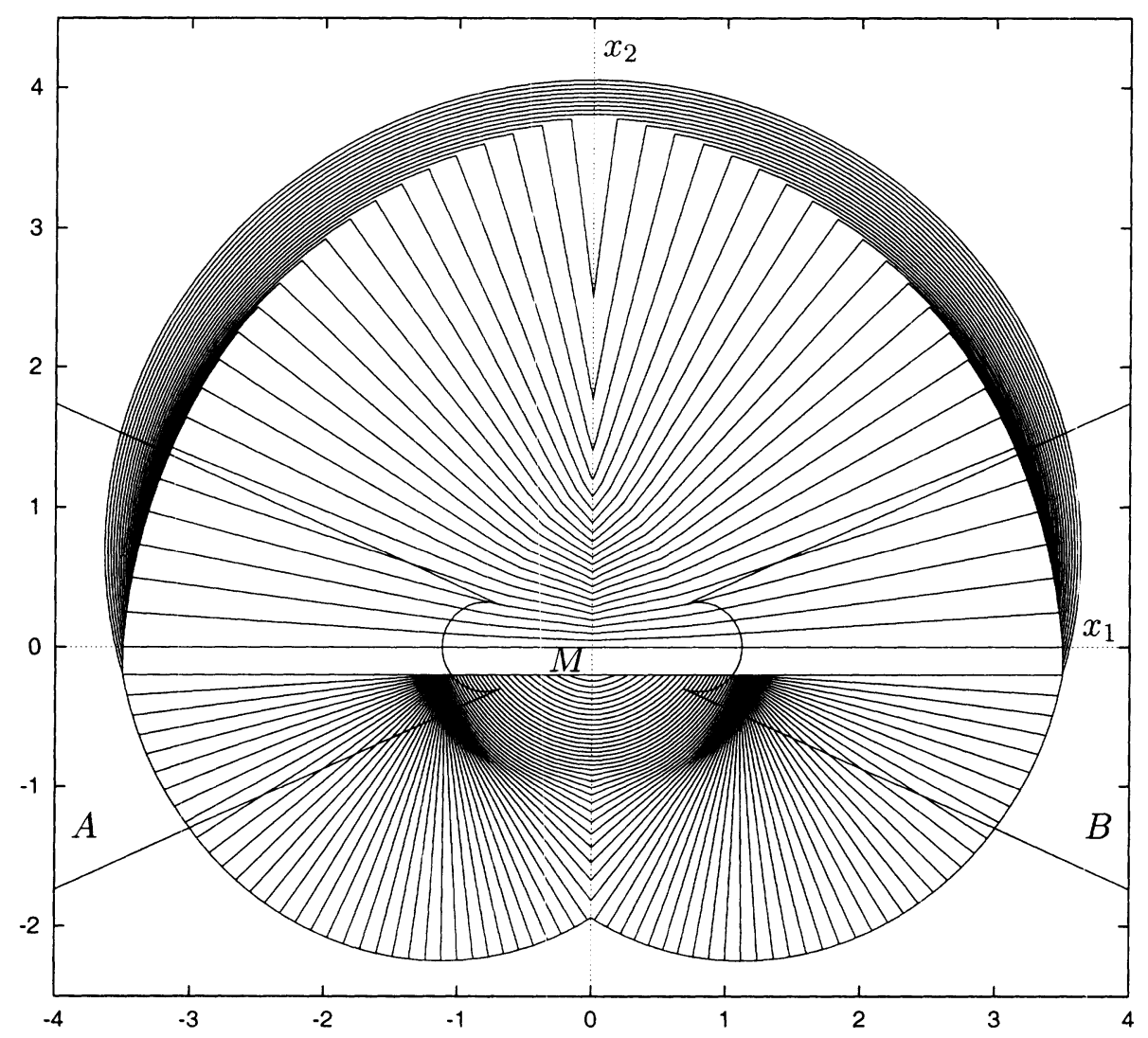

Figure 17320 upper fronts and 660 lower fronts for $w_{e}=0.4$

Figure 19 presents the computational results for $w_{e}=0.95$ and $\Delta=$ 0.005. As in the previous example, the upper barrier lines end at some moment of reverse time, and the fronts begin to envelop them. The main difference from before is the formation of a loop where the upper fronts from the two sides of the figure meet. In this example, the region within this loop (a "lagoon") is filled out entirely by the further development of the fronts, the filling out being completed at $\tau=1.68$.

An important feature of the lower part of Figure 19 is that the semipermeable curve $p^{(1), 1}$, emanating from the point $a$, intersects the right barrier which is the semipermeable curve $p^{(2), 1}$. This did not happen in the previous example. Thus the right lower fronts are confined to the right side of the curve $p^{(1), 1}$. The time of attaining the terminal set becomes infinite as the fronts approach the curve $p^{(1), 1}$. A symmetric situation occurs for the left lower fronts. All the fronts are computed until $\tau=2.4$. 


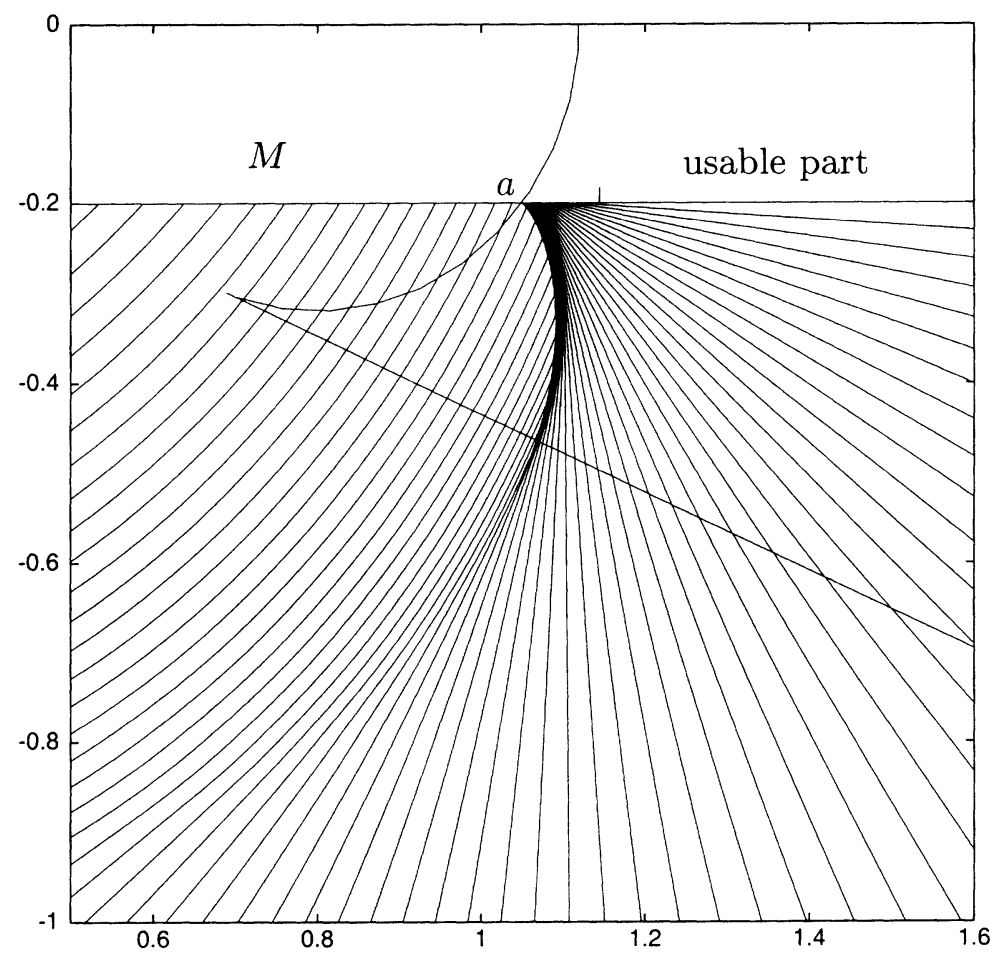

Figure 18 The accumulation of fronts near the point $a$

The following facts were found experimentally. A lagoon is generated by the upper fronts only if $w_{e} \geq 0.65$. For $w_{e} \in[0.65,1.37)$, a lagoon occurs and is completely filled by the further development of the fronts. For $w_{e} \in[1.37,1.61]$, the fronts do not fill the lagoon completely. For $w_{e}>1.61$, the lagoon disappears.

Figure 20 presents computational results for $w_{e}=1.5$ and $\Delta=0.005$. The left and right parts of the upper front meet at $\tau=2.855$. Then the computation within the lagoon begins. The fronts do not penetrate the set $D^{*}$, which is a hole inside the solvability set of player $P$, the value function being infinite for $x \in D^{*}$. The computation is done until $\tau=3.73$. The structure of the lower fronts is similar to that in the previous example.

In Figure 21, a three-dimensional graph of the value function of the Figure 20 example is presented. The axes in the horizontal plane are $x_{1}$ and $x_{2}$, and the vertical axis measures the value function. The picture shows the value function for the region of $\left(x_{1}, x_{2}\right)$ where the fronts 


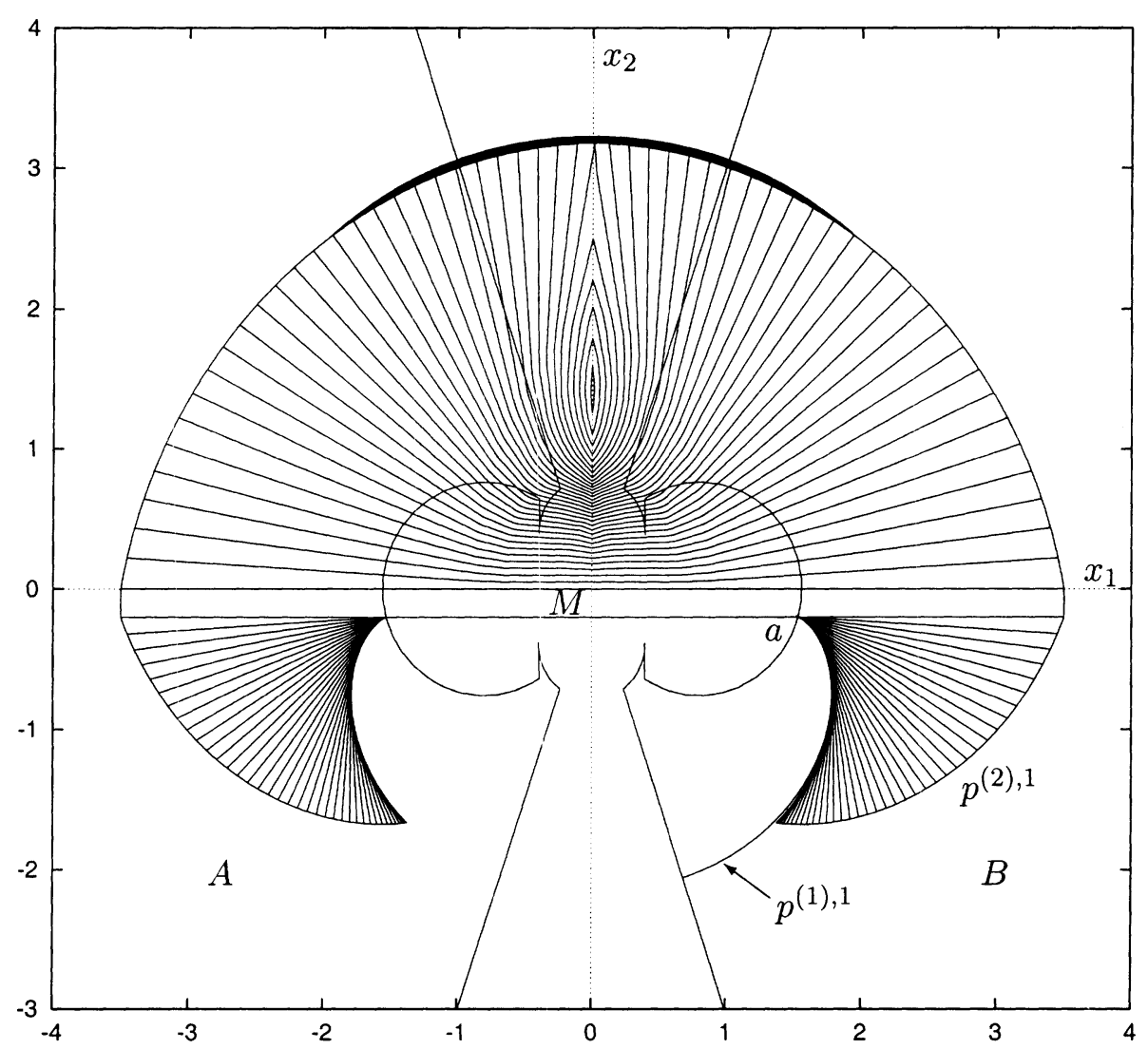

Figure 19480 upper and lower fronts for $w_{e}=0.95$ (every 10th front is plotted)

are computed. The programs for the visualization of such graphs were developed [2] by V.Averbukh and O.Pykhteev, Department of System Support, Institute of Mathematics and Mechanics, Ekaterinburg.

Further increases in the value of $w_{e}$ extend the set $D^{*}$. For example, Figure 22 gives computational results for $w_{e}=1.9$ and $\Delta=0.01$. The upper and lower fronts are computed until $\tau=8.42$ and $\tau=1.6$, respectively.

\section{CONCLUSION}

In this paper, we have studied a variant of the homicidal chauffeur differential game, under the assumption that the constraint on the control of the evader depends on the state. The two-dimensional situation allows a complete description of the families of semipermeable curves 


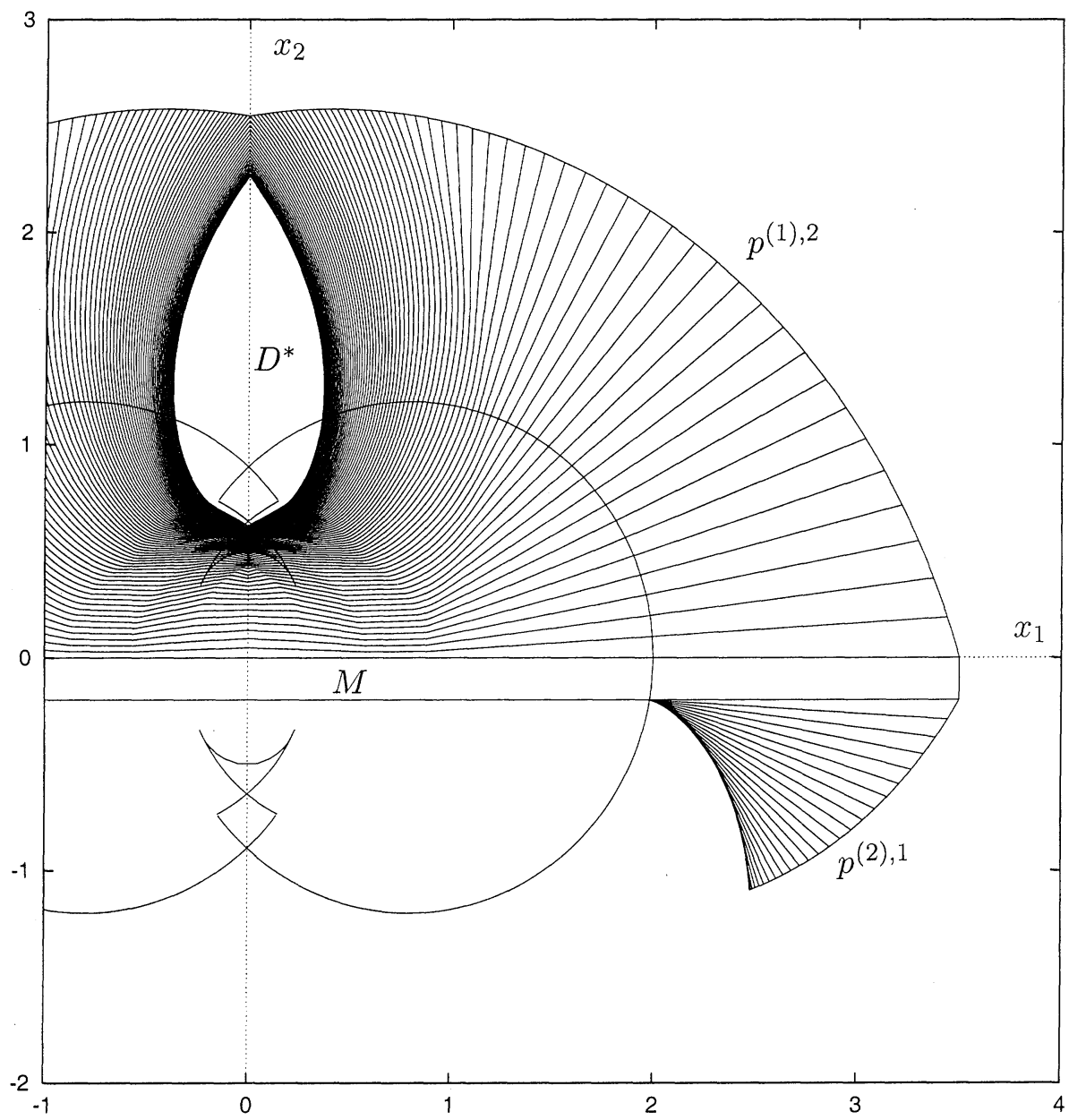

Figure 20 746 upper fronts and 340 lower fronts for $w_{e}=1.5$ (every 10th front is plotted)

that occur. The superiority sets of the evader, where semipermeable curves do not exist, are detected. Thus the presence of holes that are strictly inside the "victory domains" of the pursuer is explained. A short description of the backward procedure for the computation of level sets of the value function is also given. This procedure can be employed as a specific algorithm for two-dimensional front propagation. 


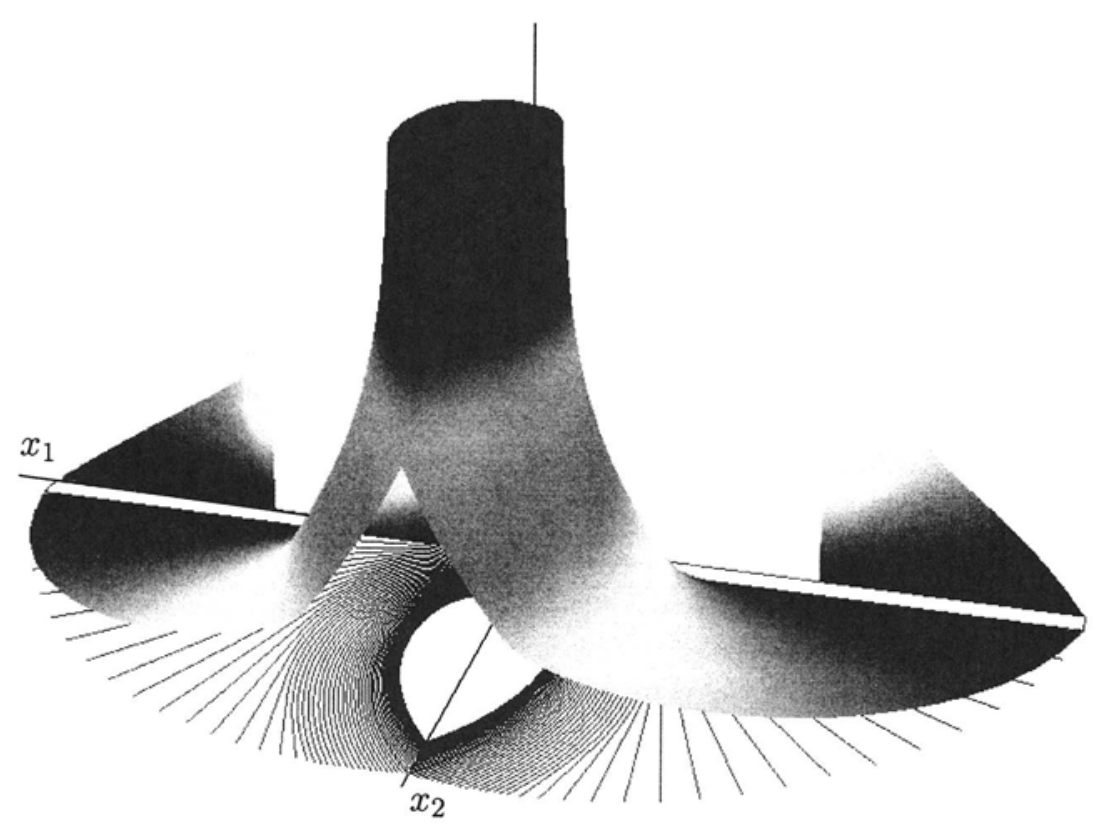

Figure 21 The graph of the value function for $w_{e}=1.5$

\section{Acknowledgments}

The authors are very grateful to M.J.D. Powell for many valuable remarks on the paper.

\section{References}

[1] J.-P. Aubin (1990), A Survey of Viability Theory, SIAM J. Control and Optimization, 28(4), pp. 749-788.

[2] V.L. Averbukh, S.S. Kumkov, V.S. Patsko, O.A. Pykhteev and D.A. Yurtaev (1999), Specialized Vizualization Systems for Differential Games, in Progress in Simulation, Modeling, Analysis and Synthesis of Modern Electrical and Electronic Devices and Systems, N.Mastorakis (Ed.), World Scientific and Engineering Society Press, pp. 301-306.

[3] P. Bernhard and B. Larrouturou (1989), Etude de la barriere pour un probleme de fuite optimale dans le plan, Rapport de Recherche, 


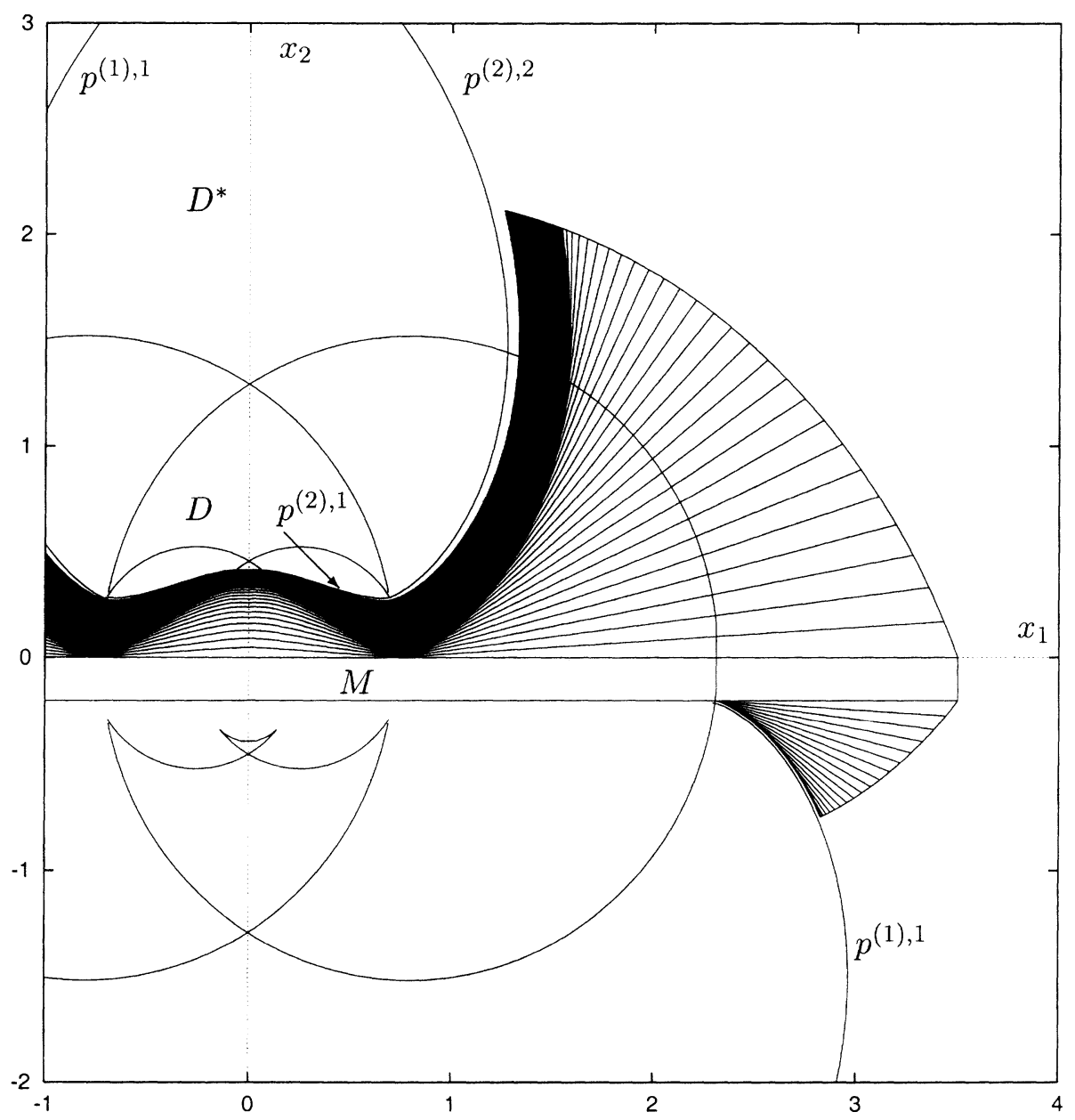

Figure 22 842 upper fronts and 160 lower fronts for $w_{e}=1.9$ (every 5 th front is plotted)

INRIA, Sophia - Antipolis.

[4] J.V. Breakwell and A.W. Merz (1970), Towards a complete solution of the homicidal chauffeur game, in Proceedings of the first international conference on the theory and applications of differential games, Amherst.

[5] P. Cardaliaguet (1997), Nonsmooth Semipermeable Barriers, Isaacs' Equation, and Application to a Differential Game with One Target and Two Players, Applied Mathematics and Optimization, 36, pp. 125-146. 
[6] P. Cardaliaguet, M. Quincampoix and P. Saint-Pierre (1995), Numerical methods for optimal control and differential games, Ceremade CNRS URA 749, University of Paris - Dauphine.

[7] R. Isaacs (1965), Differential Games, John Wiley, New York.

[8] J. Lewin and G.J. Olsder (1979), Conic Surveillance Evasion, Journal of Optimization Theory and Applications, 27(1), pp. 107-125.

[9] N.N. Krasovskii and A.I. Subbotin (1974), Positional Differential Games, Nauka, Moscow.

[10] N.N. Krasovskii and A.I. Subbotin (1988), Game-Theoretical Control Problems, Springer-Verlag, New York.

[11] A.W. Merz (1971), The Homicidal Chauffeur - a Differential Game, PhD Dissertation, Stanford University.

[12] V.S. Patsko and V.L. Turova (1995), Numerical Solution of TwoDimensional Differential Games, Preprint, Institute of Mathematics and Mechanics, Ekaterinburg.

[13] V.S. Patsko and V.L. Turova (1997), Numerical Solutions to the Minimum-Time Problem for Linear Second-Order ConflictControlled Systems, in Proceedings of the Seventh International Colloquium on Differential Equations, Plovdiv, Bulgaria, 18-23 August 1996, D.Bainov (Ed.), Utrecht, The Netherlands, pp. 327-338.

[14] V.S. Patsko and V.L. Turova (1998), Numerical study of the Homicidal Chauffeur Game, in Proceedings of the Eight International Colloquium on Differential Equations, Plovdiv, Bulgaria, 18-23 August 1997, D.Bainov (Ed.), Utrecht, The Netherlands, pp. 363-371.

[15] V.S. Patsko and V.L. Turova (1998), Homicidal Chauffeur Game. Computation of Level Sets of the Value Function, Preprints of the 8th Int. Symposium on Differential Games and Applications. Maastricht, 5-7 July, 1998, pp. 466-473. 